\title{
Caracterização e geração de combustíveis gasosos utilizando folha de coqueiro da produção do coco (Cocos nucifera)
}

\author{
Characterization and generation of gaseous fuels using coconut leaf from coconut production
}

(Cocos nucifera)

Caracterización y generación de combustibles gaseosos utilizando hoja de coco de la producción de coco (Cocos nucifera)

Recebido: 03/06/2021 | Revisado: 09/06/2021 | Aceito: 10/06/2021 | Publicado: 23/06/2021

\author{
Pedro de França Santos \\ ORCID: https://orcid.org/0000-0001-8250-2204 \\ Universidade de Pernambuco, Brasil \\ francaps@gmail.com \\ Túlio Amaral Góis \\ ORCID: https://orcid.org/0000-0001-6130-9819 \\ Universidade de Pernambuco, Brasil \\ E-mail: tuliogois20@gmail.com \\ Sérgio Peres Ramos da Silva \\ ORCID: https://orcid.org/0000-0002-2235-3507 \\ Universidade de Pernambuco, Brasil \\ E-mail: sergperes@gmail.com
}

\begin{abstract}
Resumo
Diminuir as emissões de dióxido de carbono sem afetar a oferta de energia mundial tornou a busca por novas fontes de energia uma alternativa para solucionar este problema. O reaproveitamento de resíduos agrícolas surge como uma boa alternativa. No ano de 2014 o Brasil tinha capacidade de produzir aproximadamente 5,5 milhões de cocos por dia, e com esta grande produção também se gera uma grande quantidade de resíduos. Portanto este trabalho visa apresentar um estudo do aproveitamento de resíduos da agricultura do coco (folha). Propôs-se o estudo para a utilização dessa biomassa na forma in natura e na forma de gás, obtido através da gaseificação variando o tempo de residência e a temperatura de gaseificação, para assim encontrar o gás que apresenta o maior rendimento de conversão energética. Após a caracterização das amostras, foi possível verificar que a biomassa apresenta resultado positivo para a sua utilização como uma fonte alternativa de energia. Na forma in natura o valor de PCI foi de $18,96 \mathrm{MJ}^{\mathrm{kg}} \mathrm{kg}^{-1}$. Já na gaseificação o maior valor encontrado foi de $10,46 \mathrm{MJ}^{-\mathrm{Nm}^{-3}}$.
\end{abstract}

Palavras-chave: Biomassa; Folha; Caracterização; Gaseificação.

\begin{abstract}
Reducing carbon dioxide emissions without affecting the world's energy supply has made the search for new energy sources an alternative to solve this problem. The reuse of agricultural residues appears as a good alternative. In 2014, Brazil had the capacity to produce approximately 5.5 million coconuts per day, and with this large production, a large amount of waste is also generated. Therefore, this work aims to present a study of the use of residues from coconut agriculture (leaf). A study was proposed for the use of this biomass in in natura form and in the form of gas, obtained through gasification by varying the residence time and the gasification temperature, in order to find the gas with the highest energy conversion yield. After characterization of the samples, it was possible to verify that biomass presents positive results for its use as an alternative source of energy. In the in natura form, the PCI value was $18.96 \mathrm{MJ} . \mathrm{kg}^{-1}$. In gasification, the highest value found was $10.46 \mathrm{MJ}_{\mathrm{Nm}}^{-3}$.
\end{abstract}

Keywords: Biomass; Leaf; Characterization; Gasification.

\section{Resumen}

Reducir las emisiones de dióxido de carbono sin afectar el suministro energético mundial ha hecho de la búsqueda de nuevas fuentes de energía una alternativa para resolver este problema. La reutilización de residuos agrícolas aparece como una buena alternativa. En 2014, Brasil tenía la capacidad de producir aproximadamente 5,5 millones de cocos por día, y con esta gran producción, también se genera una gran cantidad de residuos. Por tanto, este trabajo tiene como objetivo presentar un estudio del uso de residuos de la agricultura de coco (hoja). Se propuso un estudio para el aprovechamiento de esta biomasa en forma in natura y en forma de gas, obtenida mediante gasificación variando el tiempo de residencia y la temperatura de gasificación, con el fin de encontrar el gas con mayor rendimiento de 
conversión energética. Luego de la caracterización de las muestras, se pudo verificar que la biomasa presenta resultados positivos para su uso como fuente alternativa de energía. En la forma in natura, el valor de PCI fue de 18,96 MJ.kg-1. En gasificación, el valor más alto encontrado fue de 10,46 MJ.Nm ${ }^{-3}$.

Palabras clave: Biomasa; Hoja; Caracterización; Gasificación.

\section{Introdução}

No final de 2015 foi realizada em Paris a Conferência das Partes (COP-21). Esta conferência tinha como objetivo discutir e estabelecer objetivos para a diminuição das mudanças climáticas causadas pelo homem. Ao final do encontro, o Acordo de Paris foi assinado por 195 países, onde os principais pontos foram: o aumento da temperatura média global fique abaixo dos $2^{\circ} \mathrm{C}$; países ricos devem ajudar países mais pobres através de financiamento de projetos de geração de energia sustentável; o acordo vai ser revisto a cada 5 anos para a atualização das metas.

O principal motivo para a geração dos gases de efeito estufa (GHG) é a utilização de combustíveis fósseis. Isto se deve porque os combustíveis fósseis são uma fonte de energia acessível e disponível, sendo assim a maior parte do consumo de energia do mundo vem dessa fonte, o que aumenta as emissões de $\mathrm{CO}_{2}$, sendo este gás um dos responsáveis pelo efeito estufa (Patel; Zhang; Kumar, 2016).

Antes dos combustíveis fósseis se tornarem a principal fonte de energia no século 19, a biomassa era a maior fonte de energia no mundo. A Biomassa é o termo usado para toda matéria orgânica oriundas de plantas (algas, árvores e culturas) (Tanksale; Beltramini; Lu, 2010).

A biomassa é produzida através da conversão da energia solar em plantas, por meio da fotossíntese que além da energia solar também utiliza gás carbônico $\left(\mathrm{CO}_{2}\right)$ e água, sendo assim, qualquer matéria orgânica que teve energia solar armazenada em suas ligações químicas. Se a biomassa passar por um processo de produção de energia eficiente, é correto afirmar que a biomassa possui um ciclo-carbono neutro, pois todo o $\mathrm{CO}_{2}$ que foi produzido para a produção de energia foi consumido pela planta antes da sua utilização (Mckendry, 2002).

Biomassa é toda forma de planta incluindo florestas e resíduos agrícolas. Ela apresenta uma vantagem sobre os combustíveis fósseis (gás natural, carvão e petróleo), pois ela pode prover de forma contínua sua matéria prima (Ahmed et al, 2016). Atualmente, fontes renováveis têm recebido muita atenção ao redor do mundo, o uso de biomassa como fonte de energia mundial tem sido de aproximadamente de 10-14\% do total (Mckendry, 2002). Uma das maiores fontes de biomassa se encontra na forma de resíduos agrícolas.

Resíduos agrícolas incluem materiais orgânicos que foram gerados na colheita e no processamento de culturas. Estas sobras da agricultura variam de palha de arroz, talos de milho, casca de arroz, casca de coco, entre outros (Buah; Williams, 2012).

\subsection{Objetivos}

Este trabalho propõe produzir combustíveis gasosos utilizando um dos resíduos da plantação do coco, a folha.

\subsection{Objetivos específicos}

- Caracterizar físico-química do resíduo;

- Determinar energeticamente a melhor condição de gaseificação de cada biomassa;

- Variando a temperatura de gaseificação e o tempo de residência.

- Analisar a energia disponível no Nordeste. 


\section{Cultura do Coco e Resíduos}

O coqueiro (Cocos nucifera L.) é uma planta tropical muito conhecida, cultivada em áreas equatoriais e subequatoriais para produzir copra (polpa do coco), material que pode ser processado para virar óleo. No entanto o coqueiro pode prover muitos outros produtos, um deles é a água de coco, que consiste em um líquido que vem do interior do fruto verde (COSTA et al, 2015). Segundo a Organização das Nações Unidas para a Alimentação e Agricultura (FAO, 2014), o Brasil ocupa a $4^{\mathrm{a}}$ posição mundial em produção de coco no mundo, perdendo apenas para países asiáticos. Dentro do Brasil as maiores áreas plantadas concentram-se no Norte e Nordeste, onde a região nordestina é responsável por 70\% da produção nacional, sendo o total da produção brasileira de 1.973 .366 mil frutos com uma área plantada de 284.951 hectares (IBGE, 2014). Esta grande produção de coco também é responsável por uma grande geração de resíduos, é estimada uma geração de 729 mil toneladas de casca apenas no Nordeste (NUNES, 2009). Porém a casca não é a única fonte de resíduo, a folha do coqueiro também representa um problema para as fazendas. Por esta razão se torna interessante o estudo sobre o potencial energético da folha do coqueiro.

A maioria das cascas de coco e folhas do coqueiro são queimados ou descartados como lixo nas propriedades rurais produtoras de coco (Figura 1). Quando queimados produzem substâncias poluidoras do meio ambiente e quando descartados constituem meio adequado para procriação de animais peçonhentos e insetos vetores de doenças, servindo como agente poluidor do meio ambiente e de risco para a saúde dos trabalhadores rurais (Nunes; Santos; Santos, 2007).

Figura 1: Resíduos Agrícolas da Plantação de Coco.

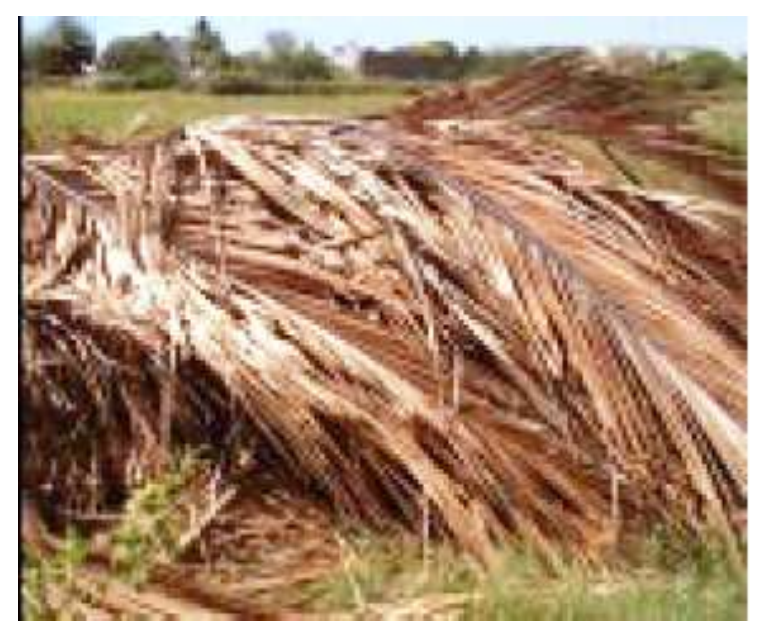

Fonte: Nunes, Santos e Santos (2007).

Em média a folha do coqueiro pesa $2,45 \mathrm{~kg}$ é produzida 12 vezes ao ano e tem a mesma quantidade é perdida nesse período. As folhas não podem ser aparadas, pois poderá afetar seu desenvolvimento e produção a de coco (Siqueira; Ribeiro; Aragão, 1994). Normalmente a folha cai seca, que é exatamente esta que fica se acumulando como lixo nas plantações. Este acúmulo é tão grande que apenas no Nordeste é estimado um valor de 595 mil toneladas de resíduos de folhas (Nunes, 2009).

Um dos motivos para uma quantidade tão grande de resíduos é seu alto custo para o reaproveitamento, o que fazem empresários desistirem desta prática (Idoeta, 2014).

Existem diversos processos em que se transforma a biomassa em energia. O objetivo desta transformação é tornar a biomassa um material para a utilização em diversas variedades, tornando-o assim economicamente viável, a Figura 2 mostra alguns desses processos. 
Figura 2: Processos de conversão da biomassa.

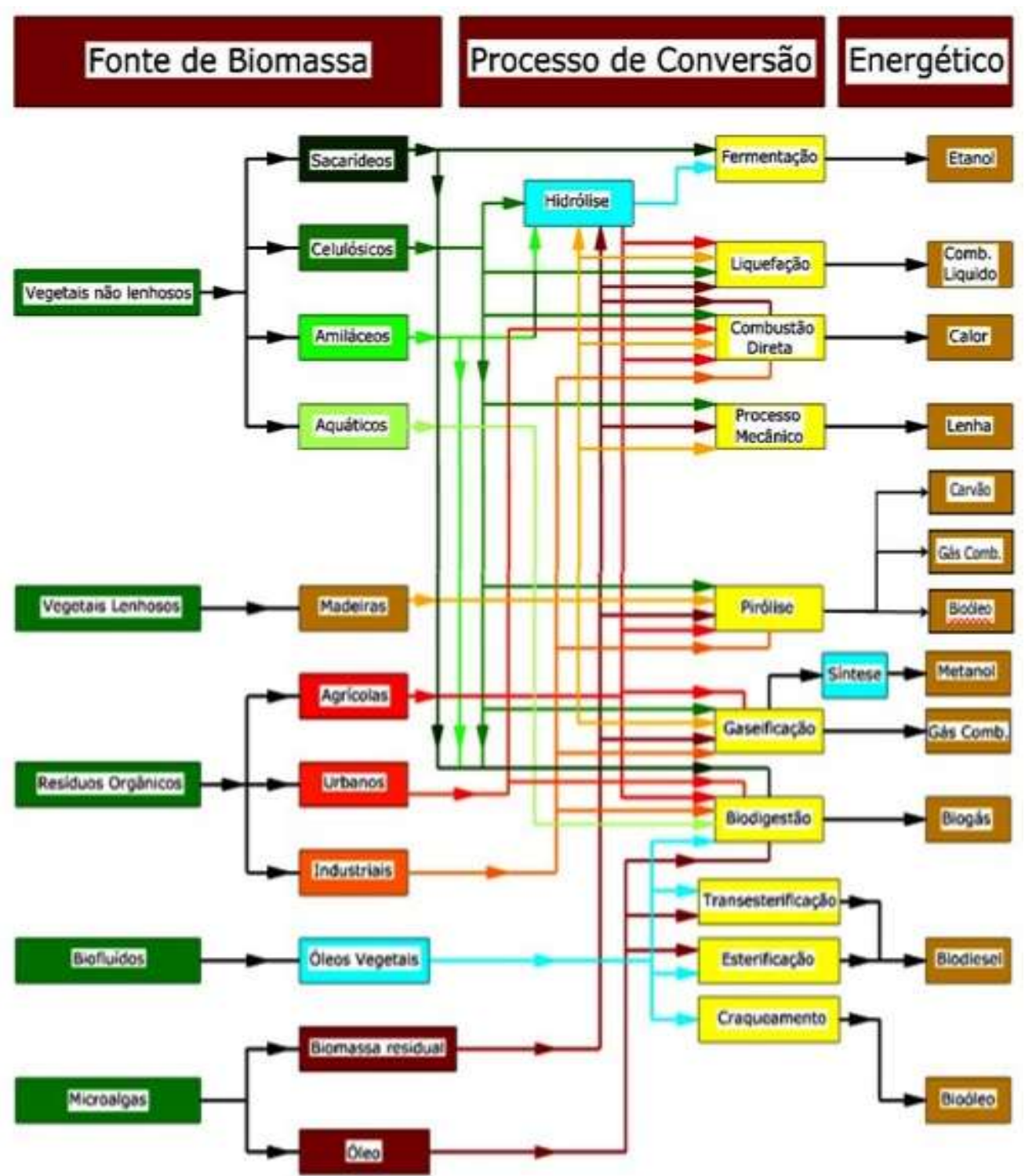

Fonte: Adaptado de Sharma et al (2013).

\section{Gaseificação}

Para este estudo foi usado o processo de gaseificação. A gaseificação é um processo de oxidação parcial em altas temperaturas de uma matéria prima carbonáceo. Além de um material para a gaseificação, é necessário para o processo um agente gaseificador como oxigênio, ar, dióxido de carbono ou uma mistura desses gases. O produto da gaseificação é uma mistura gasosa (hidrogênio, monóxido de carbono, dióxido de carbono, dióxido de carbono, metano e hidrocarbonetos de cadeia curta) conhecida como gás de síntese ou syngas. Além do produto gasoso, o processo de gaseificação deixa como subproduto as cinzas (Kaushal \& Tyagi, 2017).

Desses gases produzidos, apenas o monóxido de carbono, hidrogênio e hidrocarbonetos de cadeia curta são energeticamente ativos. Os outros gases não possuem valor energético, isso contribui na diluição do gás e abaixa o seu poder calorífico. Além destes gases, o processo de gaseificação produz o alcatrão, que apesar de ser um combustível, é um elemento indesejável, pois quando quente pode formar incrustações nas tubulações. Ao esfriar pode entupir as tubulações e os queimadores (Chaves, 2007). 
O gás de síntese pode ser aplicado de diversas maneiras como na geração de calor, queimados diretamente, ou como combustível veicular (Mckendry, 2001). Esse combustível ainda pode ser usado para a geração de energia elétrica, onde gás é queimado em sistemas de turbinas a vapor ou usado diretamente em sistemas de turbinas a gás (Yassin et al, 2009). Uma das mais importantes aplicações é para o uso em ciclo combinado. Um fluxograma para o aproveitamento foi demonstrado por Mahinpey e Gomez (2009) na Figura 3.

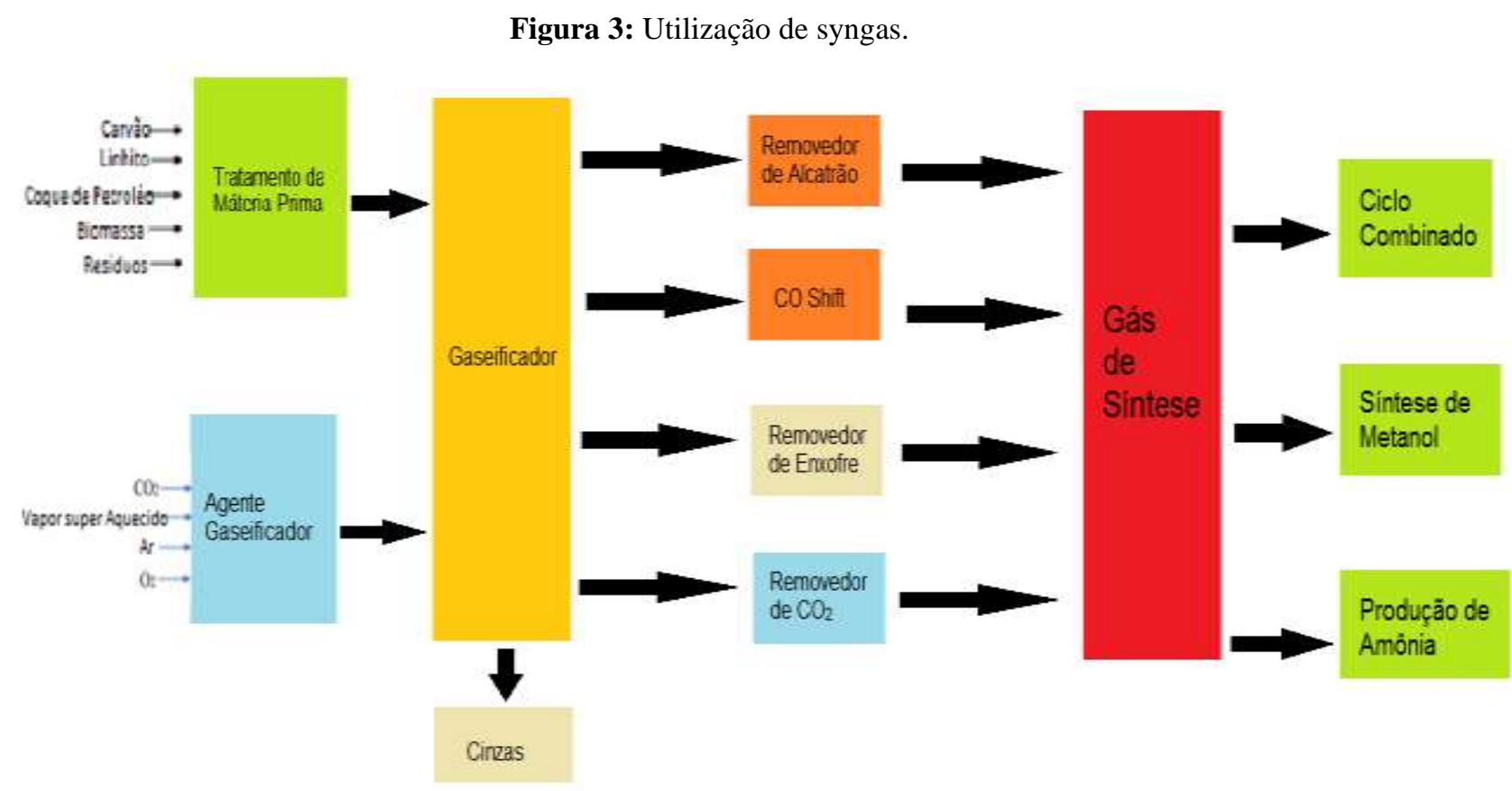

Fonte: Adaptado de Mahinpey (2009).

\section{Metodologia}

Este estudo foi realizado no Laboratório de Combustíveis e Energia (POLICOM) da Escola Politécnica da Universidade de Pernambuco (UPE). Para efeitos de estudo, os experimentos foram feitos in natura. Todos os experimentos foram feitos em triplicata.

A casca, folha e o tronco do coqueiro foram coletados na Praia do Paiva-PE. Logo após a coleta as amostras foram levadas para o POLICOM (Laboratório de Combustíveis e Energia) e então preparadas para os experimentos. Para a preparação, as biomassas foram colocadas em um moinho de facas para que tivessem uma granulometria menor uma vez que é mais adequado para a realização dos experimentos.

Segundo Estrela (2018), este trabalho possui uma metodologia qualitativa e quantitativa, já que os experimentos de caracterização são formulados por métodos próprios, enquanto que a gaseificação passa por uma análise dos resultados obtidos dos experimentos realizados.

O experimento foi realizado em um gaseificador (Figura 4) desenvolvido na própria POLICOM, com a participação dos colaboradores do laboratório. 
Figura 4: Gaseificador.

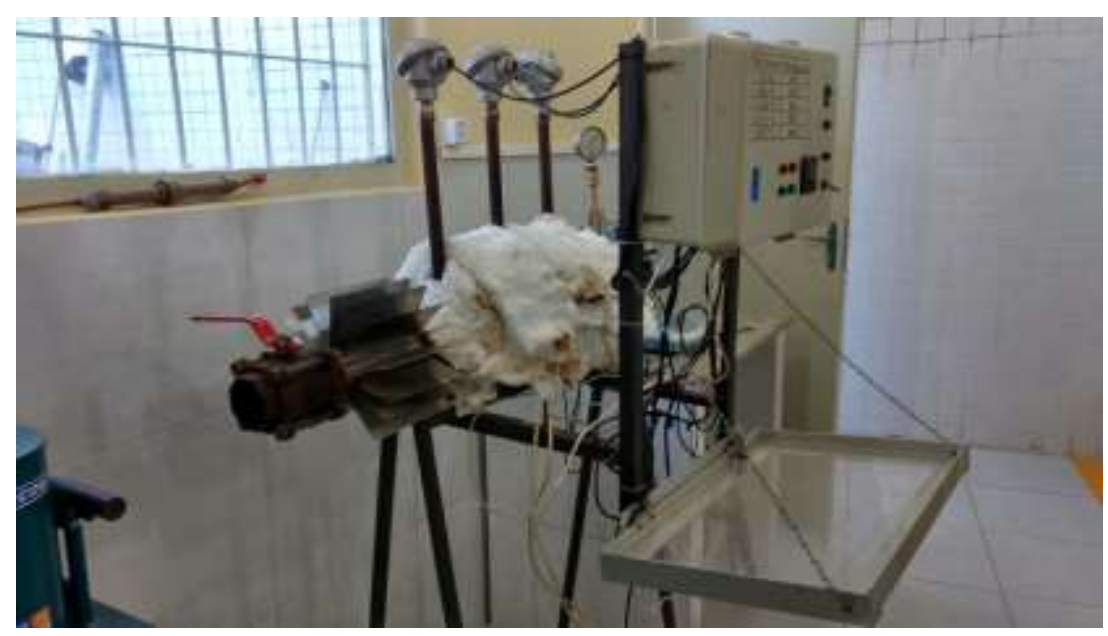

Fonte: Autores.

Através deste equipamento é possível fazer a gaseificação variando-se alguns parâmetros, tais como: temperatura, tempo de residência e massa, podendo ser a biomassa in natura, pelletizada ou em forma de grão. Este equipamento permite a coleta de gases e resíduos da gaseificação além de ser possível calcular de forma simplificada a quantidade de gás gerada em cada gaseificação.

O gaseificador é aquecido através de duas resistências de $1000 \mathrm{~W}$ cada uma e opera em regime de batelada. Esta é a tecnologia mais adequada para utilização em pequena escala, porém ela não permite acompanhar os produtos intermediários da gaseificação. No entanto esta informação não é necessária para o conhecimento dos gases finais e o cálculo do rendimento de conversão do processo, as variáveis que precisam ser conhecidas são a temperatura, tempo de residência, massa da amostra e pressão interna (utilizada para o cálculo do volume gerado) e massa final.

O gaseificador tem o desenho técnico apresentado na Figura 5. O equipamento é constituído por uma tubulação principal (1), sendo interligada por 3 ramificações (2) onde um termopar do tipo k está conectado (3). Como pode ser observada na Figura 5, uma manta isolante é utilizada com o objetivo de evitar descargas elétricas e a dissipação do calor fornecida pelas resistências (4). Na extremidade frontal do gaseificador há uma válvula esfera em aço inoxidável AISI 304 (5), por esta válvula que é feita a entrada do material a ser gaseificado. Entre a válvula de injeção e a primeira ramificação, existe um sistema de aletas (6), que é utilizado para a dissipação de calor, evitando que o operador sofra queimaduras ao trabalhar com o equipamento. No final do gaseificador é instalando um manômetro analógico (7), conectado por um sifão roscado de latão. Para que o gás permaneça enclausurado no gaseificador, foi montada uma válvula agulha (9), esta mesma válvula permite que o gás final seja recolhido após a sua abertura. Antes do gás chegar as bolsas de coleta ele passa por um filtro (10), através destas bolsas é que se torna possível levar os gases para os equipamentos que fazem as análises dos gases. Para que a biomassa seja inserida de forma segura são utilizados recipientes de aço inoxidável AISI 304 (11), de forma que o material fique na parte mais quente do equipamento. 


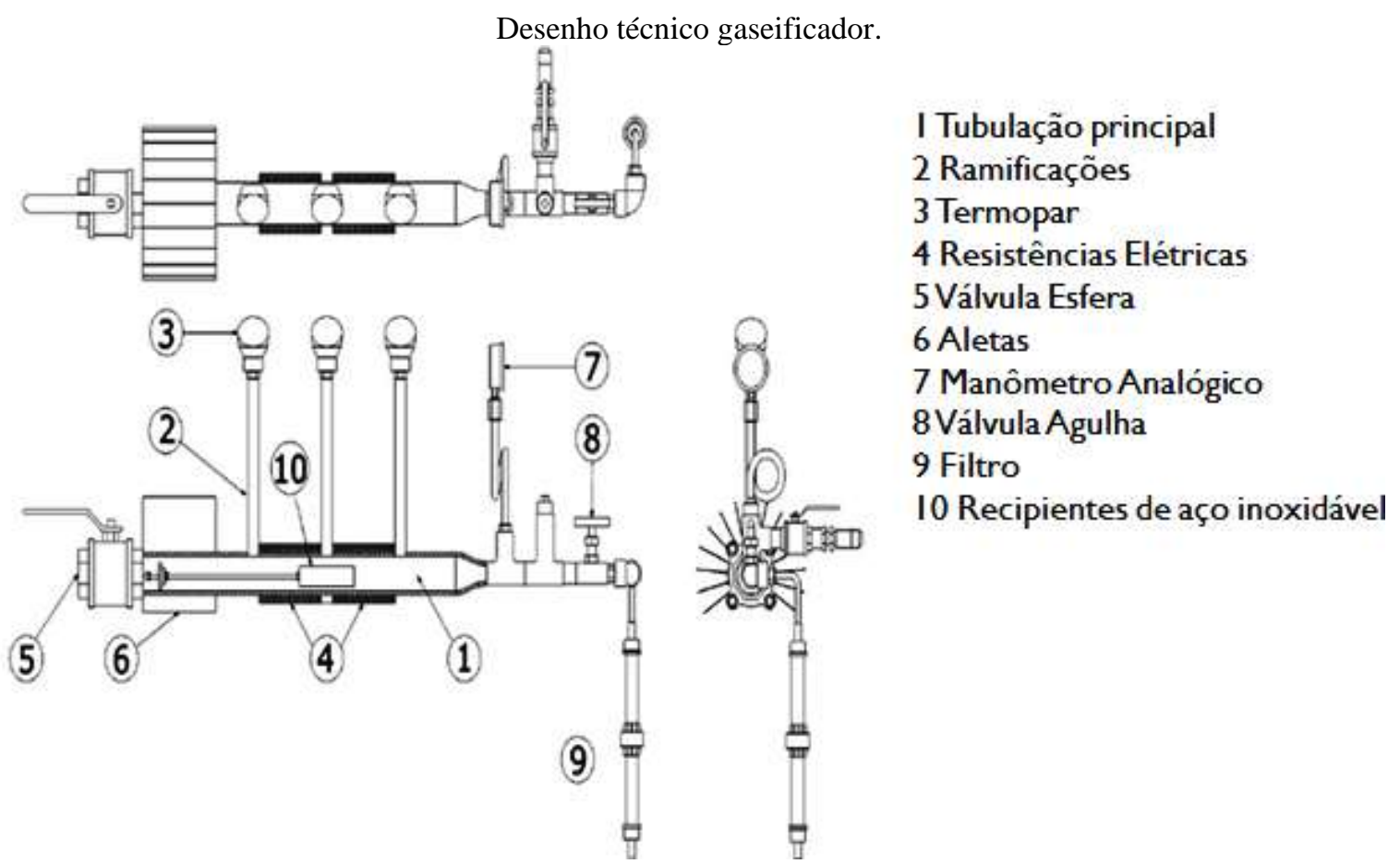

Fonte: Adaptado de Lacerda (2015).

Foi realizado o experimento seguindo uma série de procedimentos. As amostras da casca do coco, folha e tronco do coqueiro estavam em formatos de grão, todas as gaseificações foram feitas em triplicatas; a massa utilizada de biomassa foi de nove gramas.

As gaseificações foram feitas nas temperaturas de 700,800 e $900^{\circ} \mathrm{C}$ e as amostras tiveram um tempo de residência de 3, 4 e 5 minutos. O gás produzido foi colhido em uma bolsa plástica e levado para uma análise de cromatografia para que fosse encontrada a concentração dos gases produzidos.

A análise quantitativa do gás, que informa quanto de gás a biomassa é capaz de gerar por quilograma de biomassa, é possível por causa do manômetro que mostra a pressão interna do gaseificador. Para o cálculo ser possível são necessárias algumas considerações, onde é fundamental admitir que o ar (momento antes da gaseificação) e o gás (momento final da gaseificação) são gases ideais que seguem a Lei do Gás Ideal (Equação 1).

$$
\mathrm{Pv}=\mathrm{nRT}
$$

Onde ainda pode-se igualar a constante universal dos gases para os dois momentos da gaseificação seguindo a Equação 2.

$$
R=\text { constante }=\frac{P_{1}, V_{1}}{n_{1}, T_{1}}=\frac{P_{2}, V_{2}}{n_{2}, T_{2}}
$$

$\mathrm{O}$ índice 1 irá indicar o momento antes da gaseificação enquanto o índice 2 demonstra o momento após a gaseificação. Como o volume e a temperatura não variam durante o processo, o aumento de pressão é devido ao aumento do número de moles do gás (Equação 3).

$$
\frac{P_{1}}{n_{1}}=\frac{P_{2}}{n_{2}}
$$

Ao calcular o $\mathrm{n}_{2}$ é possível calcular o volume $\mathrm{V}_{2}$ pela Equação 1 que representa a Lei do Gás Ideal. 
Com o volume de gás gerado e a massa da biomassa utilizada na gaseificação sendo conhecidos pode-se calcular a capacidade de geração de gás por quilograma de biomassa utilizando a Equação 4.

$$
G=\frac{V_{2}\left(\mathrm{Nm}^{3}\right)}{m(\mathrm{~kg})}
$$

Com as informações fornecidas pelos cromatógrafos foi possível calcular o poder calorifico inferior e superior dos gases gerados. Para isso é utilizado a Tabela 1 que mostra os valores do PCI e do PCS de diversos gases. Para cálculo dos poderes caloríficos dos gases obtidos, multiplicou-se as porcentagens dos gases recolhidos pelos seus respectivos valores.

Tabela 1: Poder Calorífico de diversos gases.

\begin{tabular}{ccccccccc}
\hline Gases & $\mathbf{H}_{\mathbf{2}}$ & $\mathbf{C O}$ & $\mathbf{C O}_{\mathbf{2}}$ & $\mathbf{C H}_{\mathbf{4}}$ & $\mathbf{C}_{\mathbf{2}} \mathbf{H}_{\mathbf{6}}$ & $\mathbf{C}_{\mathbf{3}} \mathbf{H}_{\mathbf{6}}$ & $\mathbf{I C}_{\mathbf{4}} \mathbf{H}_{\mathbf{1 0}}$ & $\mathbf{N C}_{\mathbf{4}} \mathbf{H}_{\mathbf{1 0}}$ \\
\hline PCS $\left(\mathrm{MJ.Nm}^{-3}\right)$ & 12,74 & 12,63 & 0 & 39,82 & 70,29 & 93,57 & 133,12 & 134,06 \\
PCI $\left(\mathrm{MJ} . \mathrm{Nm}^{-3}\right)$ & 10,78 & 12,63 & 0 & 35,88 & 64,34 & 87,57 & 122,91 & 123,81 \\
\hline
\end{tabular}

Fonte: Autores.

Com a informação do PCI dos gases, é possível avaliar a energia total disponível de cada biomassa seguindo a equação 5 .

$$
\text { Energia Total }(M J)=P C I_{g a ̊ s}\left(M J \cdot \mathrm{Nm}^{-3}\right) x m_{\text {biomassa }}(\mathrm{kg}) x \mathrm{G}\left(\mathrm{Nm}^{3} \cdot \mathrm{kg}\right)
$$

Conhecendo o PCI do gás e da biomassa é possível avaliar o rendimento de conversão da biomassa gaseificada utilizando a equação 6. Este dado é de extrema importância, pois informa o ponto ótimo para o melhor aproveitamento energético do syngas.

$$
\eta(\%)=\frac{P C I_{g a s s}\left(M J N m^{-3}\right) x G\left(\mathrm{Nm}^{3} . \mathrm{kg}\right)}{P C I_{\text {biomassa in natura }}(\mathrm{MJ.kgg})}
$$

\section{Resultados}

A Tabela 2 mostra os resultados da caracterização da folha do coqueiro.

Tabela 2: Caracterização da biomassa.

\begin{tabular}{cc}
\hline Umidade (\%) & $\mathbf{1 0 , 6 5 \pm \mathbf { 0 , 3 7 }}$ \\
\hline Teor de cinzas (\%) (b.s.) & $6,17 \pm 3,20.10^{-4}$ \\
\hline Poder Calorífico Superior $\left(\mathbf{M J . k g}{ }^{-1}\right)$ & $19,14 \pm 0,06$ \\
\hline Poder Calorífico Inferior $\left(\mathbf{M J . k g}{ }^{-1}\right)$ & $18,96 \pm 0,07$
\end{tabular}

Fonte: Autores.

A folha do coqueiro apresenta teor de umidade baixo (10,65\%), com esta característica, esta pode ser utilizada em processos de conversão termoquímicos. Em relação ao teor de cinzas, foi encontrado um valor abaixo do indicado pela literatura para evitar que as cinzas se tornem um problema em processos de gaseificação. o PCS é a quantidade de energia liberada durante a transferência de calor, assim quanto mais energia é liberada, ou seja, maior o PCS, melhor a eficiência do 
processo. Deepak e Jnanesh (2016) encontraram para a folha do coqueiro, que também passou por um processo de secagem, um valor calórico de 15,36 MJ.kg-1 abaixo do encontrado neste trabalho, este valor do PCS é ainda maior do que diversas biomassas, como bagaço de cana (16,29 MJ.kg $\left.{ }^{-1}\right)$, espiga de milho (15,65 MJ.kg $\left.{ }^{-1}\right)$, restos de algodão $\left(17,48 \mathrm{MJ}_{\mathrm{kg}}{ }^{-1}\right)$, casca de arroz (15,29 MJ.kg-1) e diversas outras (Raveendram et al, 1995).

A Tabela 3 mostra os resultados da análise imediata.

Tabela 3: Análise Imediata.

\begin{tabular}{cc}
\hline- & Folha \\
\hline Teor de umidade (\%) & $9,45 \pm 0,36$ \\
Teor médio de & $93,51 \pm 0,23$ \\
Material volátil (\% b.s.) & \\
Teor médio de cinzas & $5,39 \pm 0,42$ \\
$(\%$ b.s. $)$ & \\
Teor de & $1,10 \pm 0,20$ \\
Carbono fixo (\%b.s.)
\end{tabular}

Fonte: Autores.

A tabela da análise termogravimétrica demonstra a média das análises feitas com o acréscimo de temperatura de 20 ${ }^{\circ} \mathrm{C} \cdot \mathrm{min}^{-1}$.

A biomassa apresenta um alto valor de material volátil $(93,51 \%)$ O que comprova que esta biomassa tem um grande potencial para seu uso como combustível. O valor das cinzas da análise imediata difere da análise do teor de cinzas porque na análise imediata é injetado oxigênio no processo, para que seja queimado o carbono que sobrou e acabou ficando nas cinzas, sendo esse carbono conhecido como carbono fixo. Como dito anteriormente a análise imediata foi utilizada biomassa granulada, para chegar nesta forma a biomassa precisou passar por um processo de secagem, por isso existe uma diferença no valor da umidade da análise imediata e do teor de umidade.

Deepak e Jnanesh (2016) realizaram a análise imediata para a folha de coqueiro, que passou por um processo de secagem, segundo os autores foi encontrado um valor de 6,90\% para umidade, 91,35\% b.s. de material volátil, 5,07\%b.s. de carbono fixo e $3,58 \%$ b.s. de teor de cinzas.

A Tabela 4 mostra a análise de fibras.

Tabela 4: Análise de Fibras.

\begin{tabular}{cc}
\hline- & Folha \\
\hline Lignina (\%b.s.) & $42,90 \pm 0,98$ \\
Celulose (\%b.s.) & $22,87 \pm 0,72$ \\
Hemicelulose (\%b.s.) & $14,65 \pm 1,12$ \\
Cinzas Insolúveis (\%b.s.) & $5,41 \pm 0,02$ \\
\hline
\end{tabular}

Fonte: Autores.

A soma da média dos itens da tabela não dá $100 \%$, esta soma é $85,83 \%$, para completar os $100 \%$ serão necessários acrescentar as porcentagens de umidade, sacarídeos, cinzas solúveis e outros. 
Conhecer o teor de fibras é um parâmetro importante uma vez que são essas que irão informar se a biomassa é eficiente na produção de combustível, pois a proporção de celulose e lignina é essencial para a escolha do processo de conversão da mesma. Para ilustrar a importância desse dado é possível afirmar que a biodegradabilidade da celulose é maior que a da lignina, com isto a produção de biogás de um biodigestor pode ser afetada (Mckendry, 2001).

A análise é feita pelo método proposto por Van Soest em 1965, que consiste no fracionamento dos componentes fibrosos, por meio de reagentes específicos, possibilitando assim uma maior precisão na estimativa do percentual de celulose, hemicelulose, lignina e cinzas insolúveis presentes na biomassa (Rodrigues, 2010).

A última análise de caracterização é a análise elementar, que é apresentada na Tabela 5.

Tabela 5: Análise Elementar.

\begin{tabular}{cc}
\hline Amostra & Folha \\
\hline N (\% b.s $)$ & $0,07 \pm 0,01$ \\
C (\%b.s) & $45,08 \pm 0,61$ \\
H (\%b.s) & $7,54 \pm 0,06$ \\
S (\%b.s) & $1,45 \pm 0,11$ \\
O (\%b.s) & $18,93 \pm 1,23$ \\
\hline
\end{tabular}

Fonte: Autores.

McKendry (2002) aponta que quanto maior a proporção de oxigênio e hidrogênio, em comparação com o carbono, o valor energético de um combustível é reduzido, devido a menor energia contida em carbono-oxigênio(C-O) e carbonohidrogênio (C-H), que em ligações carbono-carbono (C-C).

A Tabela 6 e 7 mostram os resultados obtidos no processo de gaseificação.

Tabela 6: Resultado da gaseificação.

\begin{tabular}{ccccccccc}
\hline Temp $\left({ }^{\circ} \mathbf{C}\right)$ & $\begin{array}{c}\text { Tempo } \\
(\mathbf{m i n})\end{array}$ & $\mathbf{H}_{2}(\%)$ & $\mathbf{C O}(\%)$ & $\mathbf{C H}_{4}(\%)$ & $\mathbf{C}_{2} \mathbf{H}_{\mathbf{6}}(\%)$ & $\mathbf{C}_{\mathbf{3}} \mathbf{H}_{\mathbf{8}}(\%)$ & $\mathbf{N C}_{\mathbf{4}} \mathbf{H}_{\mathbf{1 0}}(\%)$ & $\mathbf{I}_{\mathbf{4}} \mathbf{H}_{\mathbf{1 0}}(\%)$ \\
\hline $\mathbf{7 0 0}$ & 3 & $5,66 \pm 1,11$ & $18,51 \pm 0,69$ & $9,60 \pm 0,84$ & $0,53 \pm 0,53$ & $0,42 \pm 0,07$ & $0,16 \pm 0,02$ & $0,48 \pm 0,13$ \\
$\mathbf{7 0 0}$ & 4 & $13,23 \pm 0,57$ & $16,21 \pm 0,77$ & $10,40 \pm 0,50$ & $0,00 \pm 0,00$ & $0,32 \pm 0,06$ & $0,13 \pm 0,02$ & $0,23 \pm 0,03$ \\
$\mathbf{7 0 0}$ & 5 & $12,12 \pm 0,71$ & $15,65 \pm 0,47$ & $11,75 \pm 0,50$ & $0,00 \pm 0,00$ & $0,25 \pm 0,05$ & $0,03 \pm 0,03$ & $0,14 \pm 0,09$ \\
$\mathbf{8 0 0}$ & 3 & $12,66 \pm 1,79$ & $20,28 \pm 2,17$ & $12,39 \pm 0,72$ & $1,27 \pm 0,29$ & $0,46 \pm 0,10$ & 0 & 0 \\
$\mathbf{8 0 0}$ & 4 & $14,88 \pm 0,74$ & $12,48 \pm 2,32$ & $11,16 \pm 1,84$ & $1,27 \pm 0,46$ & $0,21 \pm 0,04$ & 0 & 0 \\
$\mathbf{8 0 0}$ & 5 & $14,78 \pm 1,14$ & $10,27 \pm 1,42$ & $11,83 \pm 1,46$ & $0,89 \pm 0,38$ & $0,20 \pm 0,02$ & 0 & 0 \\
$\mathbf{9 0 0}$ & 3 & $17,26 \pm 3,39$ & $6,96 \pm 0,35$ & $12,67 \pm 1,02$ & $0,99 \pm 0,07$ & $0,14 \pm 0,07$ & 0 & 0 \\
$\mathbf{9 0 0}$ & 4 & $21,16 \pm 0,81$ & $6,21 \pm 0,27$ & $15,01 \pm 0,30$ & $0,95 \pm 0,05$ & $0,17 \pm 0,01$ & 0 & 0 \\
$\mathbf{9 0 0}$ & 5 & $19,28 \pm 0,55$ & $4,94 \pm 0,27$ & $14,46 \pm 0,25$ & $1,45 \pm 0,65$ & $0,12 \pm 0,06$ & 0 & 0 \\
\hline
\end{tabular}


Tabela 7: Resultado da gaseificação.

\begin{tabular}{cccccc}
\hline $\begin{array}{c}\text { Temp } \\
\left({ }^{\circ} \mathbf{C}\right)\end{array}$ & Tempo $(\mathbf{m i n})$ & PCI $\left(\mathbf{M J} \cdot \mathbf{m}^{-\mathbf{3}}\right)$ & Carvão + cinzas $(\mathbf{\%})$ & Volume $\left(\mathbf{N m}^{\mathbf{3}} \cdot \mathbf{k g}^{-\mathbf{1}}\right)$ & $\boldsymbol{\eta}(\mathbf{\%})$ \\
\hline $\mathbf{7 0 0}$ & 3 & 7,94 & $13,66 \pm 8,63 \cdot 10^{-4}$ & $0,24 \pm 0,02$ & 10,10 \\
$\mathbf{7 0 0}$ & 4 & 7,96 & $15,40 \pm 5,23.10^{-4}$ & $0,16 \pm 2,50.10^{-3}$ & 6,19 \\
$\mathbf{7 0 0}$ & 5 & 7,96 & $14,15 \pm 2,88.10^{-3}$ & $0,19 \pm 3,23.10^{-3}$ & 6,65 \\
$\mathbf{8 0 0}$ & 3 & 10,46 & $14,71 \pm 0,01$ & $0,27 \pm 0,01$ & 15,06 \\
$\mathbf{8 0 0}$ & 4 & 9,02 & $14,46 \pm 0,01$ & 11,60 \\
$\mathbf{8 0 0}$ & 5 & 8,72 & $19,70 \pm 0,05$ & $0,24 \pm 0,01$ & 11,25 \\
$\mathbf{9 0 0}$ & 3 & 8,96 & $14,61 \pm 0,01$ & $0,24 \pm 0,01$ & 9,76 \\
$\mathbf{9 0 0}$ & 4 & 10,30 & $15,58 \pm 3,87.10^{-3}$ & $0,19 \pm 6,65.10^{-4}$ & 10,15 \\
$\mathbf{9 0 0}$ & 5 & 9,98 & $15,45 \pm 4,26.10^{-3}$ & $0,17 \pm 7,38.10^{-3}$ & 9,07 \\
\hline
\end{tabular}

Fonte: Autores.

O melhor resultado para a produção de hidrogênio acontece em $900{ }^{\circ} \mathrm{C}$ com um tempo de de residência de 4 min, ou seja, na temperatura mais alta em que foram realizados os experimentos, e no tempo de gaseificação intermediária. é possível observar que a conforme aumentamos a temperatura a concentração de hidrogênio também aumenta, e com o aumento do tempo de residência de 3 para 4 minutos há um aumento na concentração de hidrogênio, mas com o tempo de residência de 5 minutos é observado uma pequena queda em sua concentração.

O melhor resultado para a produção de monóxido de carbono acontece para as biomassas com 3 minutos de tempo de residência e $800^{\circ} \mathrm{C}$, com concentração de 20,28\% v/v. É possível observar que para a folha de coqueiro, ocorre uma diminuição na concentração de CO conforme aumentamos o tempo de residência.

O melhor resultado acontece em $900{ }^{\circ} \mathrm{C}$ para a biomassas estudada, com o tempo de residência que foi de 4 minutos sendo responsável por $15,01 \%$ da concentração do gás de síntese. A produção de metano demonstra uma melhora em sua concentração conforme é aumentada a temperatura de gaseificação. O tempo de residência não demonstra ser um fator decisivo para a produção de metano.

A folha de coqueiro apresenta a melhor situação para a produção de etano na temperatura de $900^{\circ} \mathrm{C}$ e tempo de residência de 5 min e mostra uma concentração de $1,45 \%$ v/v.

Já os gases propano e butano, não apresentam grandes quantidades, não podendo assim ser feita uma análise da produção destes.

A folha de coqueiro apresenta a melhor situação para a geração de gás, quando a temperatura é de $800^{\circ} \mathrm{C}$ e tempo de residência de 3 minutos, sendo o seu valor de $0,27 \mathrm{Nm} 3 . \mathrm{kg}^{-1}$.

Para a folha a sua melhor conversão ocorreu quando a gaseificação foi feita a $800^{\circ} \mathrm{C}$ e tempo de residência de 3 minutos, sendo o seu valor de $15,06 \%$ o melhor entre todas as situações estudadas. 


\section{Conclusões}

Ao final da realização deste trabalho, pode-se concluir que a folha do coqueiro apresenta característica satisfatória que permite o aproveitamento como combustíveis. Este aproveitamento pode ser feito tanto na forma de briquetes, na combustão direta, como na forma de gás, após o processo de gaseificação.

Utilizando na queima direta a folha de coqueiro apresentou um poder calorífico inferior de 18,96 MJ.kg-1, valor que é semelhante ao de biomassas já utilizadas para este fim.

A gaseificação destas biomassas também mostrou que a biomassa pode ser convertida para o formato de gás. A folha do coqueiro teve seu melhor valor energético de $10,46 \mathrm{MJ} . \mathrm{Nm}^{-3}$ com a gaseificação sendo realizada a $800{ }^{\circ} \mathrm{C}$ e com tempo de residência 3 minutos.

A folha de coqueiro que é capaz de gerar mais resíduo, cerca de 1.038.323 toneladas por ano, sua estimativa de geração é estimada em de 205,92 GWh.mês ${ }^{-1}$.

Como sugestões para continuação desse trabalho ficam os seguintes temas:

- $\quad$ Gaseificação em processo contínuo;

- Variar outras características da biomassa (granulometria, pellet, etc);

- $\quad$ Estudo de utilização do carvão residual;

- $\quad$ Análise econômica de uma usina que utilize syngas.

\section{Referências}

Ahmad, A. A., Norfadhila, A. Z., Farizul, H. K., Abrar, I. \& Azduwin K. (2016) Assessing the gasification performance of biomass: A review on biomass gasification process conditions, optimization and economic evaluation. Renewable and Sustainable Energy Reviews, 53, $1333-1347$.

Buah, W. K., \& Williams, P. T. (2012) Agricultural waste biomassa converted to actived carbono as a material for gold processing. Journal of Material Cycles and Waste Management, 14, 396-402.

Chaves, M. D. (2007) Gaseificação de materiais lignocelulósicos para geração de energia elétrica. Dissertação de mestrado, Universidade Federal de Lavras.

Costa, H. B., Souza, L. M., Soprani, L. C., Oliveira, B. G., Ogawa, E. M., Korres, A. M. N., Ventura, J. A. \& Romão, W. (2015) Monitoring the physicochemical degradation of coconut water using ESI-FT-ICR MS. Food Chemistry, 174, 139-146.

Deepak, K. B. \& Jnanesh, N. A. (2016) Analysis of various characteristics of coconut leaves as a biomass briquette. National Conference on Advances in Mechanical Engineering Science (NCAMES-201), 69-73.

Estrela, C. (2018). Metodologia Científica: Ciência, Ensino, Pesquisa. Editora Artes Médicas.

FAO 2014. World Production. www.faostat.org.br

IBGE. Produção Agrícola Municipal. htttp:www.sidra.ibge.gov.br/bda/pesquisa

Idoeta, P. A. (2014) Custos fazem empresários desistirem de reciclar coco em São Paulo. BBC Brasil, http://www.bbc.com/portuguese/noticias/2014/02/140207_coco_sub_reciclagem_cidades_pai

Kaushal, P. \& Tyagi, R. (2017) Advanced simulation of biomass gasification in a fluidized bed reactor using ASPEN PLUS. Reneweable Energy, 101, 629636, 2017.

Lacerda C. G. (2015) Produção de gases combustíveis utilizando o bambu em processo de gaseificação. Dissertação de mestrado.

Patel, M., Zhang, X. \& Kumar, A. (2016) Techno-economic and life cycle assessment on lignocellulosic biomass thermochemical conversion Technologies: A review. Renewable and Sustainable Energy Reviews, 53, 1486-1499.

Mahinpey, N. \& Gomez, A. (2016) A Review of gasification fundamentals and new findings: Reactors, feedstock and kinetic studies. Chemical Engineering Science. 148, 14-31.

Mckendry, P. (2002) Energy production from biomass (part 1): overview of biomass. Bioresource Technology, 83, 37-46.

Mckendry, P. (2002) Energy production from biomass (part 3): gasification technologies. Bioresource Technology 83, 53-63.

Nunes, M. U. C. (2009) Aproveitamento de Residuos do coqueiro na indústria e na agricultra. II Congresso Brasileiro de resíduos orgânicos e na agricultura.

Nunes, M. U. C., Santos, J. R. \& Santos, T. C. (2007) Tecnologia para a biodegradação da casca de coco seco e de outros residuos do coqueiro, Aracaju: EMBRAPA Tabuleiros costeiros , 5p. (Embrapa tabuleiros Costeiros. Circular técnica, 46). 
Research, Society and Development, v. 10, n. 7, e34610716783, 2021

(CC BY 4.0) | ISSN 2525-3409 | DOI: http://dx.doi.org/10.33448/rsd-v10i7.16783

Rodrigues, R. C. (2010) Métodos de análises bromatológicas de alimentos: Métodos Físicos, Químicos e Bromatológicos. Embrapa.

Sharma, B., Ingalls, R. G., Jones, C. L. \& Hkanchi, A. (2013) Biomass supply chain design and analysis: Basis overview, modeling, challenges, and future. Renewable and Sustainable Energy Review. 24, 608-627, 2013.

Siqueira, E. R., Ribeiro, F. E. \& Aragão, W. M. (1984) Melhoramento genético do coqueiro. Aracaju: EMBRAPA, 87-120.

Tanksale, A., Beltramini, J. N. \& LU, G. M. (2010) A Review of catalytic hydrogen production processes from biomass, Renewable and Sustainable Energy Reviews, 14, 166-182.

Waldheim, L. \& Nilsson, T. (2001) Heating value of gases from biomass gaseification. IEA Bioenergy Agreement subcommittee on Thermal Gasification of Biomass.

Yassin, L., Lettieri, P., Simmons, S. J. R. \& Germanà, A. (2009) A Techno-economic performance of energy-from waste fluidized bed and gasification procsses in the UK context. Chemical Engineering Journal, 146, 315-327, 2009. 